Bulletin of Mathematical Biology (1999) 61, 19-32

Article No. bulm.1998.0072

Available online at http://www.idealibrary.com on IDE $\mathbf{L}$

\title{
About Deterministic Extinction in Ratio-dependent Predator-Prey Models
}

\section{CHRISTIAN JOST, *}

Ecologie des populations et communautés, 2154, Université Paris-Sud XI,

Bât. 362, 91405 Orsay cedex, France,

and

Institut national agronomique,

Paris-Grignon,

75231 Paris cedex 05, France

E-mail: Christian.Jost@epc.u-psud.fr

\section{OVIDE ARINO}

Mathématiques appliquées,

Université de Pau et des Pays de l'Adour,

64000 Pau, France

\section{ROGER ARDITI}

Ecologie des populations et communautés, 2154, Université Paris-Sud XI,

Bât. 362, 91405 Orsay cedex, France,

and

Institut national agronomique,

Paris-Grignon,

75231 Paris cedex 05, France

Ratio-dependent predator-prey models set up a challenging issue regarding their dynamics near the origin. This is due to the fact that such models are undefined at $(0,0)$. We study the analytical behavior at $(0,0)$ for a common ratio-dependent model and demonstrate that this equilibrium can be either a saddle point or an attractor for certain trajectories. This fact has important implications concerning the global behavior of the model, for example regarding the existence of stable limit cycles. Then, we prove formally, for a general class of ratio-dependent models, that $(0,0)$ has its own basin of attraction in phase space, even when there exists a nontrivial stable or unstable equilibrium. Therefore, these models have no pathological dynamics on the axes and at the origin, contrary to what has been stated by some authors. Finally, we relate these findings to some published empirical results.

(C) 1999 Society for Mathematical Biology

*Author to whom correpondence should be addressed.

$0092-8240 / 99 / 010019+14 \quad \$ 30.00 / 0$

(C) 1999 Society for Mathematical Biology 


\section{INTRODUCTION}

Continuous predator-prey models have been studied mathematically since publication of the Lotka-Volterra equations. The principles of this model, conservation of mass and decomposition of the rates of change into birth and death processes, have remained valid until today and many theoretical ecologists adhere to these principles.

Modifications were limited to replacing the Malthusian growth function, the predator per capita consumption of prey or the predator mortality by more complex functions such as the logistic growth, Holling type I, II and III functional responses or density-dependent mortality rates.

The mentioned functional responses all depend on prey-abundance $N$ only, but soon it became clear that predator abundance $P$ can influence this function (Curds and Cockburn, 1968; Hassell and Varley, 1969; Salt, 1974) by direct interference while searching or by pseudo-interference [in the sense of Free et al. (1977)] and models were developed incorporating this effect (Hassell and Varley, 1969; DeAngelis et al., 1975; Beddington, 1975).

However, these models usually require more parameters and their analysis is complex. Therefore, they are, on one side, rarely used in applied ecology and, on the other side, have received little attention in the mathematical literature.

A simple way of incorporating predator dependence into the functional response was proposed by Arditi and Ginzburg (1989) who considered this response as a function of the ratio $N / P$.

Interesting properties of this approach have emerged that are in contrast with predictions of models where the functional response only depends on prey abundance [e.g., Arditi et al. (1991), Ginzburg and Akçakaya (1992), Arditi and Michalski (1995)]. Two principal predictions for ratio-dependent predator-prey sytems are: (1) equilibrium abundances are positively correlated along a gradient of enrichment (Arditi and Ginzburg, 1989) and (2) the 'paradox of enrichment' (Rosenzweig, 1971) either completely disappears or enrichment is linked to stability in a more complex way. However, we will not discuss here the general ecological significance of this class of models but rather study a particular mathematical feature of this model: the behavior around the point $(0,0)$ (where the models are not directly defined) and its implications on global behavior. Interesting dynamic behaviors such as deterministic extinction and multiple attractors can occur.

There are only few mathematical publications that study ratio-dependent models. Many of them use logistic-type models where density dependence in the growth equation is proportional to the ratio consumer/resource [e.g., the popular HollingTanner model (Tanner, 1975)]. However, these models do not abide by the conservation of mass rule [reproduction rate of predators is a function of the consumption rate, Ginzburg (1998)]. We are rather interested in ratio-dependent models that respect this conservation of mass (or energy) as an important aspect of ecological modelling. This further reduces the available literature on this class of models. Cosner (1996) developed floor and ceiling functions to understand the behavior of 
complex systems that include temporal variability, and ratio-dependent formulations proved to be more adapted to this kind of study. Beretta and Kuang (1998) studied the influence of delays on the stability behavior of the non-trivial equilibrium.

Freedman and Mathsen (1993) studied conditions for persistence of a specific ratio-dependent predator-prey model. They restricted their analysis to parameter values that ensure that the equilibrium $(0,0)$ behaves like a saddle point. They based this restriction on the assertion that attractivity of this trivial equilibrium is possible only with parameter values for which the predator abundance $P(t)$ increases without bound as a function of time. In this paper, we will show that this assertion is erroneous and we will reanalyse the general stability behavior of a typical ratiodependent model around the equilibrium $(0,0)$. Furthermore, we will give a formal proof (for a general ratio-dependent model) that this point can become attractive for all initial conditions sufficiently close to the predator axis, while the non-trivial equilibrium remains either locally stable or becomes unstable. This gives rise to global behaviors that range from global attractivity of the non-trivial equilibrium, coexistence of two different attractors (each with its own basin of attraction) to global attractivity of the equilibrium $(0,0)$.

Extinction is a frequent outcome in simple laboratory predator-prey systems (Gause, 1935; Luckinbill, 1973) and biologists had to modify conditions in order to obtain (cyclic) coexistence [e.g., spatial heterogeneities (Huffaker, 1958) or viscous medium to slow down the predators (Veilleux, 1979)]. Since traditional predatorprey models predict cyclic dynamics, extinction has been explained as the result of stochasticity occurring when the trajectories come close to the axes. In this paper we show that, for some region in the parameter space of a ratio-dependent model, multiple attractors can appear, one of them being the origin. Therefore, extinction can be explained as a simple deterministic process.

\section{The Model ANd Its Equilibria}

A predator-prey system that incorporates conservation of mass and division of population rates of change into birth and death processes has the following canonical form:

$$
\begin{aligned}
& \frac{d N}{d t}=f(N) N-g(N, P) P \\
& \frac{d P}{d t}=e g(N, P) P-\mu P
\end{aligned}
$$

with prey abundance $N(t)$ and predator abundance $P(t)$, conversion efficiency $e$ and predator death rate $\mu$. We will use the traditional logistic form for the growth function $f$ with maximal growth rate $r$ and carrying capacity $K$ :

$$
f(N)=r\left(1-\frac{N}{K}\right)
$$


The functional response $g$ (prey eaten per predator per unit of time), that in general depends on both prey and predator density, will be considered as a (bounded) function of the ratio prey per predator,

$$
g:=g\left(\frac{N}{P}\right)=\frac{\alpha N / P}{1+\alpha h N / P}=\frac{\alpha N}{P+\alpha h N} \quad \forall(N, P) \in[0,+\infty)^{2} \backslash(0,0)
$$

with total attack-rate $\alpha$ and handling time $h$. Note that the second equality is strictly correct only for $P>0$. In the case of $P=0$ and $N>0$ we can define $g(N, 0):=1 / h$ (the limit of $g(x)$ for $x \rightarrow \infty$ ).

In a first step we simplify this model by non-dimensionalisation. Let

$$
\begin{aligned}
& \hat{N}=\frac{\alpha h N}{e K}, \quad \hat{P}=\frac{\alpha h P}{e^{2} K}, \quad R=\frac{r h}{e}, \\
& Q=\frac{h \mu}{e}, \quad S=\frac{\alpha h}{e} \quad \text { and } \quad \hat{t}=\frac{e t}{h} .
\end{aligned}
$$

In these new variables the system becomes

$$
\begin{aligned}
& \frac{d \hat{N}}{d \hat{t}}=R\left(1-\frac{\hat{N}}{S}\right) \hat{N}-\frac{S \hat{N}}{\hat{P}+S \hat{N}} \hat{P} \\
& \frac{d \hat{P}}{d \hat{t}}=\frac{S \hat{N}}{\hat{P}+S \hat{N}} \hat{P}-Q \hat{P}
\end{aligned}
$$

with initial conditions $\hat{N}(0)=n_{0}, \hat{P}(0)=p_{0}$. For simplicity we will not write the hat $\left(^{(}\right)$in the rest of this paper.

This system has at most three equilibria in the positive quadrant: $(0,0),(S, 0)$ and a non-trivial equilibrium $\left(n^{\star}, p^{\star}\right)$ with

$$
\begin{aligned}
n^{\star} & =\frac{S(R+(Q-1) S)}{R} \\
p^{\star} & =\frac{S(1-Q)}{Q} n^{\star} .
\end{aligned}
$$

A simple calculation shows that $n^{\star}$ is positive for all $S<R /(1-Q)$, which implies $Q<1$ and therefore ensures the positivity of $p^{\star}$.

To see why $(0,0)$ is indeed an equilibrium (despite the fact that $g$ is undefined in that case) note that for any $g$ that is a non-negative bounded function in its domain (such as (3)) the right-hand sides of system (1), (2) become 0 at this point, which is the definition of an equilibrium (boundedness of $g$ is a sufficient condition, but not a necessary one).

Figure 1 shows the possible isoclines of the system. For $S>R$, the prey isocline is a humped curve through the origin and the point $(S, 0)$. For $S<R$, the denominator 

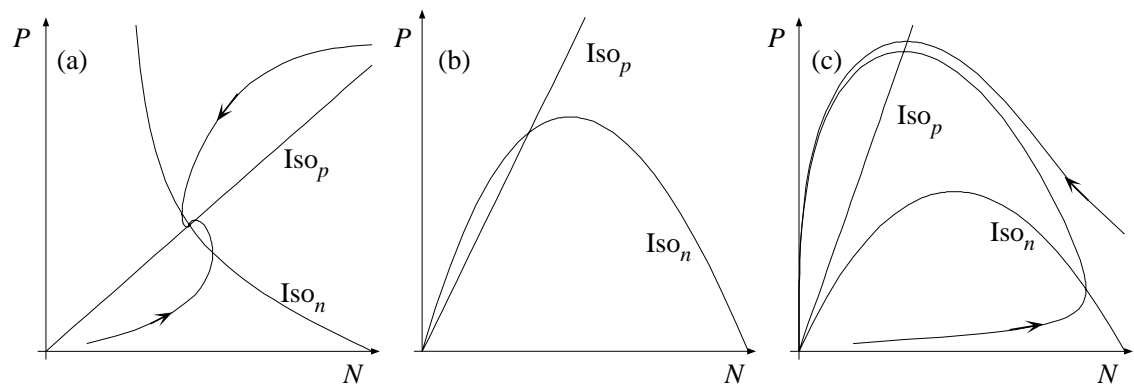

Figure 1. The three general types of isoclines that can occur: (a) the non-trivial equilibrium is stable and $(0,0)$ behaves like a saddle point $(R=0.5, Q=0.3, S=0.4)$; (b) both equilibria can be attractive or repelling, creating dynamics that are illustrated in Figs 2-5; (c) the equilibrium $(0,0)$ is globally attractive $(R=0.5, Q=0.79, S=3.0)$. The lines with arrows are examples of trajectories, Iso $n$ is the prey isocline and Iso $_{p}$ the predator isocline.

of the prey isocline can become 0 for some $N \in(0, S)$. The part of the isocline that remains in the positive quadrant becomes in this case a strictly monotonically descending curve through the point $(S, 0)$. The predator isocline is always a straight line through the origin. See Arditi and Ginzburg (1989) for more details. While the cases of Fig. 1(a) and (c) do not raise mathematical difficulties, the case of Fig. 1(b) presents interesting and unexpected mathematical properties that will be studied below.

\section{Stability OF THE EQUilibria}

The community matrix (Jacobian at the equilibrium) at the point $(S, 0)$ is

$$
\left[\begin{array}{cc}
-R & -1 \\
0 & 1-Q
\end{array}\right]
$$

and therefore, if the non-trivial equilibrium exists ( $\Longrightarrow Q<1)$, this point is always a saddle point.

The community matrix at $\left(n^{\star}, p^{\star}\right)$ has the form

$$
\left[\begin{array}{cc}
-R+S-Q^{2} S & -Q^{2} \\
(Q-1)^{2} S & (Q-1) Q
\end{array}\right]
$$

Applying the Routh-Hurwitz criterion shows that this equilibrium is stable whenever

$$
S<\min \left\{\frac{R}{1-Q}, \frac{Q-Q^{2}+R}{1-Q^{2}}\right\} .
$$

Note that, if

$$
\frac{R}{1-Q}<\frac{Q-Q^{2}+R}{1-Q^{2}}(\Longleftrightarrow R+Q<1)
$$


then the non-trivial equilibrium is always stable (if it exists). This is possible with two types of isoclines, Fig. 1(a) and (b). The case of Fig. 1(b) together with this condition (allowing arbitrarily low stable equilibrium densities of both prey and predator) is particularly interesting in the context of biological control where the interest is in non-trivial stable equilibria with $n^{\star} \ll S$. The non-trivial equilibrium in Fig. 1(a) is also always stable (independently of the above criterion), because its existence ensures that $Q<1$, therefore, if $S<R$, then $S$ also fulfills criterion (6). However, this case is less interesting because it requires high predator densities to keep the prey density low.

At the equilibrium $(0,0)$ the community matrix cannot be calculated directly because the ratio $N / P$ is not defined at this point. To understand the stability behavior of this point we must expand it on a whole axis by studying the transformed systems $(N / P, P)$ and $(N, P / N)$. Setting $L:=N / P$, then we have the system

$$
\begin{aligned}
& \frac{d L}{d t}=L\left(R+Q-\frac{R}{S} L P\right)-S \frac{L(1+L)}{1+S L} \\
& \frac{d P}{d t}=S \frac{L}{1+S L}-Q P .
\end{aligned}
$$

There are two equilibria on the $L$-axis, $(0,0)$ and $\left(\frac{S-Q-R}{S(Q+R-1)}, 0\right) .(0,0)$ is a saddle point for $S<Q+R$ (eigenvalues of the community matrix are $-Q$ and $Q+R-S$ ), otherwise it is attractive. The latter equilibrium has the eigenvalues

$$
\lambda_{1}=\frac{S(1-Q)-R}{S-1}, \quad \lambda_{2}=\frac{S Q+R-S+Q+S R-(R+Q)^{2}}{S-1}
$$

and it is unstable whenever a non-trivial equilibrium exists.

Proof. Let $S<1$. If the non-trivial equilibrium exists $(S<R /(1-Q))$ then $\lambda_{1}>0$, therefore the equilibrium is unstable.

Now let $S>1$. The existence of the non-trivial equilibrium ensures in this case that $R+Q>1$. Furthermore, $\frac{S-Q-R}{S(Q+R-1)}$ must be positive to be of interest, therefore

$$
S>Q+R
$$

and

$$
\begin{aligned}
\lambda_{2} & =\frac{1}{S-1}\left(S(Q-1)+(Q+R)-(R+Q)^{2}+S R\right. \\
& \stackrel{(7)}{>} \frac{1}{S-1}((Q+R)(Q-1+1-R-Q)+S R \\
& =\frac{1}{S-1}(R(S-(Q+R)) \stackrel{(7)}{>} 0 .
\end{aligned}
$$

This equilibrium is therefore unstable. 
Finally, we need the stability behavior of $(0,0)$ for the system $(N, M)$ with $M:=P / N$,

$$
\begin{aligned}
\frac{d N}{d t} & =N\left(R-\frac{N R}{S}-\frac{K S}{K+S}\right) \\
\frac{d M}{d t} & =\frac{M(S(N R-(Q+R-1) S)+M(N R+S(S-Q-R)))}{S(M+S)} .
\end{aligned}
$$

The community matrix at $(0,0)$ has the eigenvalues $\lambda_{1}=1-R-Q, \lambda_{2}=R$ and the point $(0,0)$ is therefore always unstable.

Summarizing we can conclude for the original system $(N, P)$ that for $S<Q+R$ the equilibrium $(0,0)$ behaves like a saddle point. For $S>Q+R$ we have seen that the system $(N / P, P)$ has an attractive equilibrium at its origin $(0,0)$. Interpreted in the original state variables $N$ and $P$ this point can only be attained by a trajectory for which ' $N$ goes faster to 0 than $P$ '. Below, we will discuss the existence of such trajectories. Freedman and Mathsen (1993), who studied in their paper the same model (4) and (5), excluded the latter case $(S>Q+R)$ from their persistence analysis of ratio-dependent models by stating (p. 823) that 'this implies that there are solutions $(N(t), P(t)) \longrightarrow(0,+\infty)$ as $t \longrightarrow \infty$ '. The following proposition proves that this statement is erroneous.

Proposition 3.1. The system of equations (4), (5) is ultimately bounded with some bound independent of the initial values.

Proof. Let $b, c>0$ such that $\frac{(R+b)^{2} S}{4 R}<c$ (for any $b$, such a $c$ can be found).

$$
\begin{aligned}
& \frac{(R+b)^{2} S}{4 R}<c \\
\Leftrightarrow & (R+b)^{2}-4 \frac{R}{S} c<0 \\
\Leftrightarrow & 0<\frac{R}{S} N^{2}-N(R+b)+c \quad \forall N .
\end{aligned}
$$

Therefore we have

$$
\begin{aligned}
\frac{d}{d t}(N+P) & =R N-\frac{R}{S} N^{2}-Q P \\
& \stackrel{(8)}{<}-b N-Q P+c \\
& <-d(N+P)+c
\end{aligned}
$$

with $d:=\min (b, Q)$. So we can conclude that $\lim _{t \rightarrow \infty} \sup (N(t)+P(t)) \leq \frac{c}{d}$. Note that we have $N(t)+P(t) \leq \max \left(N(0)+P(0), \frac{c}{d}\right), \forall t \geq 0$. 
Freedman and Mathsen (1993) also point out that a general ratio-dependent model can pose definition problems on the predator axis. However, if the functional response is restricted to being positive and bounded (two properties not contested in ecology and implicit in the model studied here), then (1) and (2) are perfectly well defined on the whole positive quadrant $[0,+\infty)^{2} \backslash(0,0)$, and the analysis in this paper shows that the behavior at $(0,0)$ has nothing abnormal that would justify its exclusion.

If the non-trivial equilibrium were unstable and the point $(0,0)$ a saddle point, then we could construct easily a positive invariant set that contains these two equilibria and apply the Poincaré-Bendixson theorem to prove the existence of a limit cycle. However, the following proposition holds:

Proposition 3.2. For $S<Q+R$ the non-trivial equilibrium (if it exists) is locally stable.

This means that, if the non-trivial equilibrium is unstable, then $S>Q+R$, implying, as shown earlier, that $(0,0)$ is not a saddle point. This complicates considerably the construction of the positive invariant set required to apply the Poincaré-Bendixson theorem. We have not found such a set but do not exclude that it can exist.

Proof of proposition. (a) For $R+Q<1$ we have already seen above that all existing non-trivial equilibria are stable.

(b) For $R+Q>1$, we have

$$
\begin{aligned}
& R=R\left(1-Q^{2}+Q^{2}\right) \stackrel{R>1-Q}{>} R\left(1-Q^{2}\right)+(1-Q) Q^{2} \\
\Longrightarrow & R>(1-Q)\left(R+Q^{2}+R Q\right) \\
\Longrightarrow & \frac{R}{1-Q}>(Q+R)(1+Q)-Q \\
\Longrightarrow & \frac{R+Q(1-Q)}{(1-Q)(1+Q)}>Q+R>S
\end{aligned}
$$

and, according to criterion (6), the non-trivial equilibrium is stable.

Getz (1984) gave a proof of existence of a stable limit cycle for a ratio-dependent model that only differed from the model used here by its prey growth function $[a r /(b N+r)-c$ instead of $r(1-N / K)]$. He did not study rigorously the behavior at $(0,0)$, simply stating that the isocline graph 'demonstrates' that it is a saddle point (as required by the Poincaré-Bendixson theorem, since the origin is part of the positive invariant set that he constructed). However, the general analysis in the next section applies also to his system and it shows that $(0,0)$ can become attractive. His graphical interpretation is therefore incorrect. It can be seen numerically that there are cases for which $(0,0)$ becomes globally attractive instead of having a stable limit cycle around the non-trivial equilibrium (as in Fig. 5). 


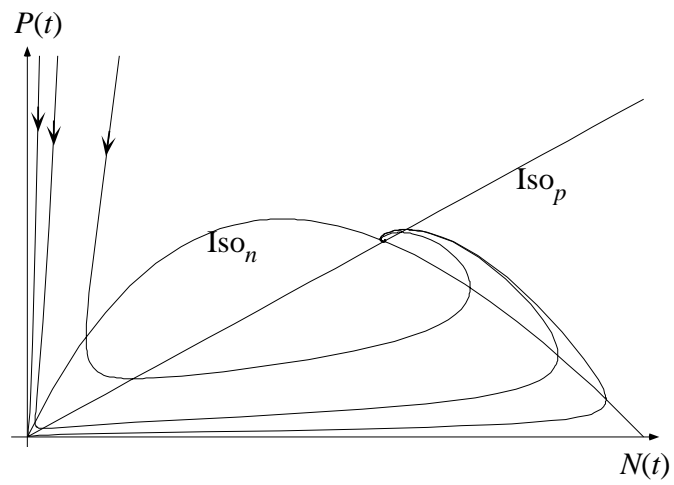

Figure 2. The non-trivial equilibrium is a global attractor and $(0,0)$ behaves like a saddle point, $S<Q+R$. Parameter values are $R=0.5, Q=0.79, S=1.0$.

\section{4. (0, 0) AS An Attractor}

So far we have only shown that the equilibrium $(0,0)$ can be attractive for trajectories where ' $N$ goes faster to 0 than $P$ ', but we do not know yet whether this type of trajectory really exists. In this section we will give a formal proof for this. This proof will be given for any growth function $f$ and any functional response $g$ in the general form of system (4), (5),

$$
\begin{aligned}
& \frac{d N}{d t}=f(N) N-g(N / P) P \\
& \frac{d P}{d t}=g(N / P) P-Q P,
\end{aligned}
$$

with $f$ and $g$ having the following properties:

- $f$ and $g$ are continuous in $R^{+}$and both functions are bounded

- $f(N)<f(0) \quad \forall N>0$

- $g(0)=0, g^{\prime}(v)$ exists and is positive for any $v \geq 0$.

Proposition 4.1. Assume $f(0)<g^{\prime}(0)-Q$ [i.e. $Q+R<S$, in our system (4), (5)]. Then, any trajectory for which $n_{0}$ is sufficiently small compared to $p_{0}$ converges to the point $(0,0)$.

Note that this proposition is a generalisation of a recent result by Kuang and Beretta (1998).

Proof. Consider the system $(N, L)$ with $L:=\frac{N}{P}$,

$$
\begin{aligned}
\frac{d N}{d t} & =f(N) N-g(L) \frac{N}{L} \\
\frac{d L}{d t} & =f(N) L-(1+L) g(L)+Q L .
\end{aligned}
$$




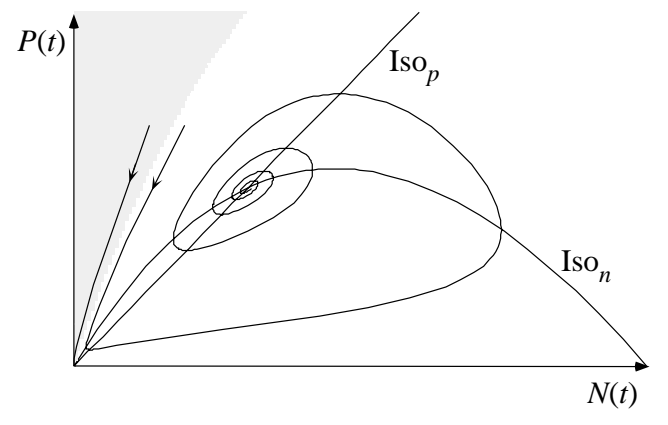

Figure 3. The non-trivial equilibrium is locally stable, but $(0,0)$ becomes also attractive, $S>Q+R$. The white area is the basin of attraction of the non-trivial equilibrium, the gray area is the one of equilibrium $(0,0)$. Parameter values are $R=0.5, Q=0.79, S=1.66$.

For $L>0$ we have

$$
\frac{d}{d t} L<f(0) L-(1+L) g(L)+Q L \stackrel{L g(L)>0}{<} L\left(f(0)-\frac{g(L)}{L}+Q\right) .
$$

Because of our assumption $f(0)<g^{\prime}(0)-Q$ we have for any $\epsilon \in\left(0, g^{\prime}(0)-Q-\right.$ $f(0))$ the stronger inequality

$$
f(0)<g^{\prime}(0)-Q-\epsilon
$$

Since $\lim _{L \rightarrow 0} \frac{g(L)}{L}=g^{\prime}(0)$ there exists some $\eta>0$ such that

$$
\left|\frac{g(L)}{L}-g^{\prime}(0)\right|<\epsilon \quad \forall 0<L<\eta .
$$

We can now conclude that

$$
\begin{array}{rlrl}
f(0)-\frac{g(L)}{L}+Q \stackrel{(9)}{<} g^{\prime}(0)-\frac{g(L)}{L}-\epsilon \stackrel{(10)}{<} 0 & & \forall 0<L<\eta \\
\Longrightarrow \frac{d}{d t} L<0 & \forall 0<L<\eta .
\end{array}
$$

Therefore, if there is some $t_{0}$ with $L\left(t_{0}\right) \leq \eta$, then $L(t) \leq \eta \forall t \geq t_{0}$ and $\frac{d}{d t} L<0$

$$
\Longrightarrow \lim _{t \rightarrow \infty} L(t)=0 .
$$

On this basis, we can further conclude that

$$
\frac{d}{d t} N \leq N\left(f(0)-\frac{g(L)}{L}\right) \stackrel{(11)}{<} 0 \quad \forall t \geq t_{0} .
$$


Therefore $\lim _{t \rightarrow \infty} N(t)=0$. Finally, consider the equation $\frac{d}{d t} P=P(g(L)-Q)$, since $L(t) \rightarrow 0$ and $g(0)=0$ there is some $t_{\omega}$ such that

$$
\begin{gathered}
\frac{d}{d t} P<0 \quad \forall t>t_{\omega} \\
\Longrightarrow \lim _{t \rightarrow \infty} P(t)=0 .
\end{gathered}
$$

This proves the proposition.

The condition $Q+R<S$ is possible with isoclines as shown in Fig. 1(b) and (c). Examples of trajectories converging to the origin are shown in Figs 35. The numerical simulations of the trajectories in these figures were done using Mathematica with the built-in high order adaptive step size procedure (the accuracy goal had to be set higher than the default value to avoid numerical problems close to the origin).

\section{Discussion}

We saw that the equilibrium $(0,0)$ can behave in several ways depending on parameter values. The following sequence of figures illustrates these behaviors by steadily increasing parameter $S$ while keeping parameters $R$ and $Q$ at fixed values. Figure 2 illustrates the case for which it is a saddle point. All trajectories converge to the non-trivial stable equilibrium independently of the initial conditions (this equilibrium is therefore a global attractor). Freedman and Mathsen (1993) derived for this case conditions that ensure persistence of the predator-prey system. Figure 3 shows the case of having two attractive equilibria, each with its own basin of attraction. The two basins were determined numerically by overlaying the phase space with a small-scale grid, taking each grid point as the initial value and determining whether the simulation ends in $(0,0)$ or in the non-trivial equilibrium. There must be a separatrix between these two basins. Figure 4 shows again a case with two basins of attraction, but the non-trivial equilibrium is now unstable and we have a stable limit cycle. As was shown in the previous section we cannot use the Poincaré-Bendixson theorem to prove the existence of this stable limit cycle because the construction of a positive invariant set would require knowledge of the analytic form of the separatrix. This figure also shows that the limit cycles will be very sensitive to stochastic influences: random perturbations to the populations occurring while the cycle is not far from the separatrix can bring the trajectory into the basin of attraction of $(0,0)$, thereby causing extinction. Figure 5 shows the case when $(0,0)$ becomes attractive for all positive initial conditions except the non-trivial equilibrium itself. There is no formal proof of this global attractivity, and several trials with Dulac's criterion failed. Further increase of parameter $S$ will make the non-trivial equilibrium disappear and $(0,0)$ becomes (trivially) globally attractive 


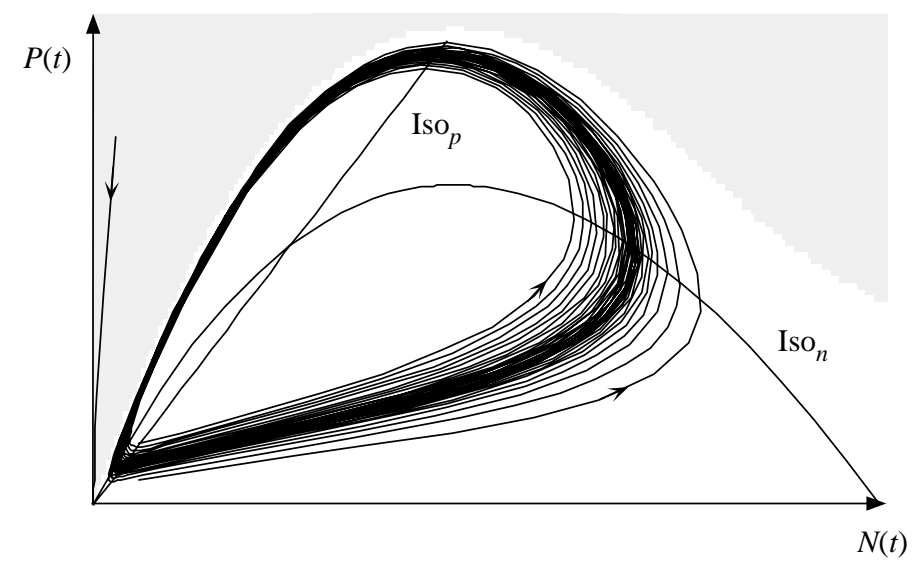

Figure 4 . The non-trivial equilibrium is unstable and $(0,0)$ becomes attractive, $S>Q+R$. There are two attractors, a stable limit cycle and $(0,0)$. Parameter values are $R=0.5, Q=$ $0.79, S=1.78$.

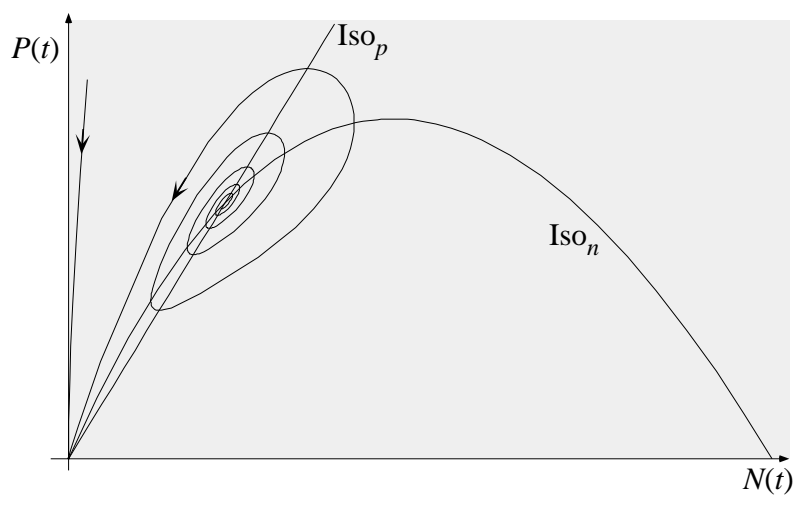

Figure 5. The equilibrium $(0,0)$ is a global attractor, $S>Q+R$. Parameter values are $R=0.5, Q=0.79, S=1.85$. There is no formal proof for the global attractivity.

[Fig. 1(c)]. The present mathematical analysis establishes that a general class of ratio-dependent models have well defined dynamics on the axes and at the origin.

Extinction of one or both populations in predator-prey systems has occupied ecologists since the classic experiments of Gause (1935), who tried to reproduce in the laboratory the cycles predicted by the Lotka-Volterra predator-prey equations. However, instead of the desired coexistence, the most frequent result was that the populations (Paramecium sp. preyed upon by Didinium nasutum) went extinct either immediately or after a couple of oscillations. Other researchers encountered the same problem [e.g., Huffaker (1958), Luckinbill (1973)]. By thickening the medium to reduce the mobility of the predator, Luckinbill (1973) repeatedly obtained several predator-prey oscillations before extinction and Veilleux (1979) refined this technique to have finally sustained cycles without extinction. He also 
did extensive experiments for various initial conditions and detected two basins of attraction (his Fig. 11) that are similar to those in our Fig. 3. Since the classical predator-prey systems like Lotka-Volterra or more complex ones with logistic growth and Holling type II functional responses cannot show deterministic extinction, these results have usually been explained by demographic stochasticity: limit cycles bring the populations very close to 0 during the cycle and small stochasticities suffice to cause extinction. The model studied here can explain the extinction as a deterministic result, with no need for stochasticity. The simultaneous existence of an unstable non-trivial equilibrium and an attractive trivial equilibrium $(0,0)$ extends the behaviors of this model from extinction after one simple oscillation, as briefly described by Arditi and Berryman (1991), to extinction after a number of oscillations. Furthermore, the technique of thickening the medium to stabilise the predator-prey interaction (Luckinbill, 1973; Veilleux, 1979) can be interpreted as reducing the attack rate $\alpha$ (Harrison, 1995) which, in the present ratio-dependent model, has a stabilising effect. By varying this parameter, the whole spectrum of observed behaviors (stable coexistence, sustained oscillations, extinction after several cycles, immediate extinction) can be predicted, as illustrated by Figs 2-5.

\section{ACKNOWLEDGEMENTS}

We thank Lev Ginzburg for emphasising repeatedly the ecological interest of understanding extinction in predator-prey systems. This research was supported by the Swiss National Science Foundation and by the French CNRS.

\section{REFERENCES}

Arditi, R. and A. A. Berryman (1991). The biological control paradox. Trends in Ecology and Evolution 6, 32 .

Arditi, R. and L. R. Ginzburg (1989). Coupling in predator-prey dynamics: ratiodependence. J. Theoret. Biol. 139, 311-326.

Arditi, R., L. R. Ginzburg and H. R. Akçakaya (1991). Variation in plankton densities among lakes: a case for ratio-dependent predation models. Amer. Naturalist 138, 1287-1296.

Arditi, R. and J. Michalski (1995). Nonlinear food web models and their responses to increased basal productivity, in Food Webs: Integrations of Patterns and Dynamics, G. A. Polis and K. O. Winemiller (Eds), London: Chapman and Hall, pp. 122-133.

Beddington, J. R. (1975). Mutual interference between parasites or predators and its effect on searching efficiency. J. Animal Ecol. 44, 331-340.

Beretta, A. and Y. Kuang (1998). Global analyses in some delayed ratio-dependent predatorprey sytems. Nonlinear Analysis, Theory, Methods \& Applications 32, 381-408.

Cosner, C. (1996). Variability, vagueness and comparison methods for ecological models. Bull. Math. Biol. 58, 207-246. 
Curds, C. R. and A. Cockburn (1968). Studies on the growth and feeding of Tetrahymena pyriformis in axenic and monoxenic culture. J. Gen. Microbiol. 54, 343-358.

DeAngelis, D. L., R. A. Goldstein and R. V. O'Neill (1975). A model for trophic interactions. Ecology 56, 881-892.

Free, C. A., J. R. Beddington and J. H. Lawton (1977). On the inadequacy of simple models of mutual interference for parasitism and predation. J. Animal Ecol. 46, 543-554.

Freedman, H. I. and R. M. Mathsen (1993). Persistence in predator-prey systems with ratio-dependent predator influence. Bull. Math. Biol. 55, 817-827.

Gause, G. F. (1935). Experimental demonstrations of Volterra's periodic oscillations in the numbers of animals. Brit. J. Exp. Biol. 12, 44-48.

Getz, W. M. (1984). Population dynamics: a per capita resource approach. J. Theoret. Biol. 180, 623-643.

Ginzburg, L. R. (1998). Assuming reproduction to be a function of consumption raises doubts about some popular predator-prey models. J. Animal Ecol. 67, 325-327.

Ginzburg, L. R. and H. R. Akçakaya (1992). Consequences of ratio-dependent predation for steady-state properties of ecosystems. Ecology 73, 1536-1543.

Harrison, G. W. (1995). Comparing predator-prey models to Luckinbill's experiment with Didinium and Paramecium. Ecology 76, 157-174.

Hassell, M. P. and G. C. Varley (1969). New inductive population model for insect parasites and its bearing on biological control. Nature 223, 1133-1137.

Huffaker, C. B. (1958). Experimental studies on predation: dispersion factors and predatorprey oscillations. Hilgardia 27, 343-383.

Kuang, Y. and E. Beretta (1998). Global qualitative analysis of a ratio-dependent predatorprey system. J. Math. Biol. 36, 389-406.

Luckinbill, L. S. (1973). Coexistence in laboratory populations of Paramecium aurelia and its predator Didinium nasutum. Ecology 54, 1320-1327.

Rosenzweig, M. L. (1971). Paradox of enrichment: destabilization of exploitation ecosystems in ecological time. Science 171, 385-387.

Salt, G. W. (1974). Predator and prey densities as controls of the rate of capture by the predator Didinium nasutum. Ecology 55, 434-439.

Tanner, J. T. (1975). The stability and the intrinsic growth rates of prey and predator populations. Ecology 56, 855-867.

Veilleux, B. G. (1979). An analysis of the predatory interaction between Paramecium and Didinium. J. Animal Ecol. 48, 787-803. 